Meta

Journal des traducteurs

Translators' Journal

\title{
Le traducteur littéraire : un marieur empathique de cultures
}

\section{Françoise Wuilmart}

Volume 35, numéro 1, mars 1990

Actes du colloque international " La traduction proligère "

URI : https://id.erudit.org/iderudit/004621ar

DOI : https://doi.org/10.7202/004621ar

Aller au sommaire du numéro

Éditeur(s)

Les Presses de l'Université de Montréal

ISSN

0026-0452 (imprimé)

1492-1421 (numérique)

Découvrir la revue

Citer cet article

Wuilmart, F. (1990). Le traducteur littéraire : un marieur empathique de cultures. Meta, 35(1), 236-242. https://doi.org/10.7202/004621ar d'utilisation que vous pouvez consulter en ligne.

https://apropos.erudit.org/fr/usagers/politique-dutilisation/ 


\section{LE TRADUCTEUR LITTÉRAIRE : UN MARIEUR EMPATHIQUE DE CULTURES}

FRANÇOISE WUILMART

Institut Lucien-Cooremans, Bruxelles, Belgique

Je constate que je suis seule à aborder le thème de la traduction littéraire.

Il est vrai qu'elle est souvent considérée comme un oiseau rare, raison pour laquelle peut-être on m'a logée aux côtés d'un représentant des paradis artificiels. Pourtant certains collègues, hier, ont déjà, consciemment ou inconsciemment, énoncé quelques vérités qui étaient valables surtout dans le cas de la traduction littéraire et que je répéterai aujourd'hui en d'autres termes.

Quoi qu'il en soit, s'il est une traduction proligère, c'est bien la traduction littéraire. Et en ce qui me concerne, le philosophe que je traduis depuis des années est précisément aussi un auteur dont l'écriture, souvent poétique, est essentiellement proligère. C'est pour cette double raison que j'ai décidé de traverser l'Atlantique, car il me semblait que dans ce contexte j'avais quelque chose d'important à vous dire.

Les problèmes spécifiques de la traduction littéraire sont multiples, mais il en est un qui me semble crucial. C'est, hélas, le prince trop souvent oublié de cette discipline, quand il n'est pas carrément banni par certaines théories actuelles: l'empathie. Ce phénomène étant intimement lié à la personnalité de l'auteur et à celle du traducteur, je vous ferai part ici d'une expérience purement personnelle, du vécu d'une traduction, me basant sur un exemple très concret, mais révélateur, de relation qui peut, qui devrait se créer entre l'écrivain et son re-créateur. Dans un premier temps, je me vois donc contrainte de vous présenter brièvement mon activité de traductrice.

Depuis près de vingt ans je me suis spécialisée dans la traduction du philosophe allemand Ernst Bloch, plus particulièrement de son cuvre maîtresse, Le Principe Espérance, qui comprend trois volumes d'environ six cents pages chacun. Cette fréquentation presque quotidienne et prolongée du grand penseur allemand ainsi que la pratique journalière de la traduction m'ont inspiré certaines réflexions sur l'opération traduisante. L'un de ses aspects, l'empathie donc, se rattache au problème, absolument essentiel en traduction littéraire, du passage d'une langue à une autre, autrement dit, d'une culture à une autre, et soulève la question brûlante de savoir si un contenu dissocié de sa forme (la langue de départ) et introduit dans une autre forme (la langue d'arrivée) ne perd pas, au cours de ce processus d'aliénation et d'écorchement vif, la plus grand partie de sa substance.

Je voudrais rappeler d'emblée la distinction très nette qu'il convient de faire entre le traducteur littéraire et le traducteur de textes techniques ou scientifiques. Les textes scientifiques et techniques appartiennent au discours d'intention objective, analytique ou descriptif et ne laissent par conséquent à la personnalité de l'auteur que très peu d'espace. Il est attendu de l'auteur qu'il s'efface autant que possible, et la priorité est donnée au phénomène et à son approche scientifique et donc neutre. Le texte littéraire est au contraire un texte d'auteur, il baigne en plein dans la subjectivité et est le résultat de l'approche d'une part artistique, de l'autre psycho-physiologique d'un monde qui nous apparaît 
précisément à travers les lunettes d'un individu. Ce monde particulier du texte littéraire a une couleur, une ambiance, un ton, un style; c'est celui de Kafka, ou de Proust, ou de Dostoïevski, et la griffe des maîtres se retrouve jusque dans les moindres détails. Cette griffe, cette signature imprègne la syntaxe, le rythme, le choix des mots, mais elle flotte aussi entre les lignes; elle est pour ainsi dire l'âme insaisissable du texte, qui se trouve à la fois partout et nulle part à chaque page. C'est à cette âme que le traducteur littéraire est confronté et doit être sensible. Mais il y a pire : c'est cette âme qu'il lui incombe de transposer, autrement dit de traduire.

La thèse que j'avance et que je défends pour avoir pu la vérifier à maintes reprises depuis de nombreuses années est que le traducteur littéraire ne pourra s'acquitter de cette tâche avec bonheur qu'à la condition qu'il y ait entre lui et l'auteur une certaine empathie.

Ce terme, assez peu usuel sans doute, évoque le mot sympathie et finalement s'en rapproche aussi par le sens. Le Petit Robert en donne la définition suivante: «faculté de s'identifier à quelqu'un, de ressentir ce qu'il ressent». Pour exprimer la chose de façon plus banale, nous pourrions dire qu'il s'agit pour le traducteur de se trouver sur la même longueur d'onde que l'auteur. Ce qui ne veut pas dire qu'il doive partager ses idées, politiques ou autres, car l'empathie se réfère à quelque chose de plus vaste et de plus profond, qui se laisse peut-être rendre au mieux par les termes de tempérament ou de nature profonde. Tel était d'ailleurs l'avis du poète allemand Stefan George, que je cite maintenant: «Il m'est impossible de traduire ce qui ne correspond pas à ma nature profonde.» Cette affirmation n'est d'ailleurs pas sans rappeler l'attitude de l'interprète musical : il est rare, par exemple, qu'un pianiste qui trouve dans l'interprétation d'une Polonaise de Chopin l'occasion de déverser sur le clavier son propre lyrisme, interprète avec un égal bonheur et une égale conviction les phrases baroques et tortueusement géométriques d'un J. S. Bach; sans doute y arriverait-il après un long travail d'application, ce qui n'empêche que son interprétation d'une cuvre de Bach demeurerait sans doute extérieure, un peu comme s'il l'avait apprise par cour et en tout cas sans la ressentir. Pour le traducteur litteraire aussi, il s'agit avant tout de ressentir le texte de départ, faute de quoi il ne pourra trouver le ton juste, la tonalité générale à mettre à la clé de son texte, faute de quoi il risque de rendre d'un bout à l'autre sur le ton majeur, par exemple, ce que l'auteur avait composé en mineur.

Pour arriver à ce ton juste, la voie rationnelle est inadéquate. Ce n'est pas avec la raison, par le chemin de l'abstraction que l'on ressent les choses. Or, un texte littéraire, dont la manifestation extrême est la poésie, cherche comme toute œuvre d'art à interpeller bien autre chose que la raison. Dans ce cas-ci la précision est donc d'une tout autre nature que celle du texte scientifique ou technique.

Tout d'abord au niveau lexical: placé dans le contexte littéraire, le mot se teinte d'une certaine coloration et sa connotation dans cette sphère particulière est parfois plus importante que son sens premier. Le mot littéraire ou poétique est comparable à une tache de couleur en peinture: un même rouge peut avoir des allures bien différentes selon qu'il est entouré de brun et d'orange, ou de blanc et de jaune, selon qu'il apparaît dans les fameux drapés d'un Van Eyck ou dans un tableau de Matisse.

Au niveau syntaxique: la phrase d'auteur est souvent une composition presque musicale dans laquelle le rythme et la sonorité jouent un rôle essentiel. Cette phrase s'inscrit à son tour dans le contexte plus vaste du paragraphe et du chapitre, eux aussi minutieusement agencés pour produire ou renforcer tel ou tel effet, créer telle ou telle ambiance, refléter tel ou tel point de vue.

Le traducteur littéraire se voit donc confronté à une double difficulté: premièrement être sensible à cette âme particulière du texte, née de tous ces moyens mis en cuvre par l'auteur, deuxièmement la recréer pour ainsi dire dans un autre matériau. 
Le traducteur est certes un lecteur privilégié, ou devrait l'être. En effet, il est sans doute le seul (mis à part l'exégète) à saisir à fond toute la portée d'un texte, puisque sa tâche consiste d'abord à refaire en sens inverse, à partir du texte original, le chemin parcouru par l'auteur, à remonter les méandres de sa pensée pour découvrir finalement la véritable clé du travail, celle qui lui permettra de décoder, de décrypter le texte jusque dans ses moindres détails : cette clé, c'est celle qui ouvre la porte de l'univers de l'auteur, constituée de ses multiples planètes: psychique, émotionnelle, affective, conceptuelle, esthétique, culturelle, etc. Or, s'appliquer à une telle tâche de prospection, aller à la rencontre par un chemin souvent ardu et lent, très lent, d'une personnalité avec laquelle on n'a pas d'atomes crochus est tout simplement infaisable. En revanche, le pied devient léger dès qu'au bout de chemin se tient quelqu'un que l'on aime; et à ce propos, je vous rappelle la célèbre phrase de Valery Larbaud : «Dis-moi qui tu traduis et je te dirai qui tu es.»

Pour illustrer de façon tout à fait concrète ce concept d'empathie entre auteur et traducteur, je voudrais vous en démontrer l'importance à la lumière d'une expérience personnelle.

L'auteur que je traduis est un philosophe. Mais c'est aussi un très grand styliste chez qui les mots clés et le style ont avant tout une fonction hautement suggestive et poétique. Il y a adéquation parfaite entre la langue d'Ernst Bloch et sa pensée.

Premièrement: penseur du matérialisme dialectique, il décrit un monde en devenir dans lequel rien n'est achevé, où tout est fragmentaire, à l'état de tendance et de latence, où la fin des fins, pour la résumer ainsi, n'apparaît que de façon suggestive et voilée, dans les symboles et les archétypes par exemple, bref, où l'Essence n'est pas encore révélée à elle-même. Parallèlement, il forge des mots (tâche rendue aisée grâce à la très grande souplesse de la langue allemande) qui cernent de façon adéquate ce phénomène imprécis d'éclosion progressive, en substantivant par exemple des infinitifs et en mettant ainsi tout l'accent sur le faire, le poïein, plutôt que sur le résultat abstrait exprimé par le substantif.

Deuxièmement: il y a dans le monde tel qu'il est conçu par Bloch une énergie, une vitalité qui s'impose depuis le début de l'histoire et perce ici et là à des niveaux différents, bref une intention qui ne cesse de ressurgir; cette force transparaît non seulement dans le choix des vocables blochiens, mais aussi dans le style vigoureux et corsé.

Troisièmement: le ton de Bloch a souvent été comparé à celui d'un prophète, et même d'un prophète en colère. Son texte est empreint d'un souffle presque biblique, et cette comparaison n'est pas gratuite: Bloch a hérité de l'espérance messianique et l'a sécularisée. C'est ce souffle qui détermine souvent le rythme du texte.

Quatrièmement: si dans son éthique Bloch préconise l'attitude critique et circonspecte du détective qui repère les lieux avant de s'y engager, bref, s'il est le disciple de Marx en cela qu'il ne conçoit pas de changement valable s'il ne s'appuie sur une analyse concrète du milieu historique et naturel, attitude qu'il appelle le courant froid du marxisme, il est surtout le penseur du courant chaud (ce sont ses propres termes), c'est-à-dire de l'enthousiasme, de la foi dans le but, sans lesquels, dira-t-il, un plan, aussi minutieusement élaboré soit-il, finira dans les tiroirs, tournera au schématisme et aux phrases creuses et s'étiolera. Ce courant chaud parcourt les trois volumes du Principe Espérance comme une sève ou comme un flot qui jamais ne tarit et est perceptible à tous les niveaux sémantiques.

Cinquièmement: dans un monde placé aujourd'hui sous le signe de la raison scientifique et technique, Bloch réhabilite l'Imaginaire. La conscience anticipante est le premier berceau du Nouveau et de tout bouleversement. Bloch passe donc en revue toutes les manifestations de cette fonction imaginaire, dans les domaines aussi bien artistiques que médicaux, religieux, sociaux, etc. La place que l'Imaginaire occupe dans son œuvre est immense et est à l'origine d'un style particulièrement haut en couleur et poétique. 
Sixièmement et enfin: pour des raisons philosophiques sur lesquelles je ne peux m'étendre ici, Bloch considère que la musique est le plus élevé de tous les arts et, parallèlement, tout son Principe Espérance est composé, bien plus qu'écrit, selon des structures musicales : tantôt celle de la fugue, tantôt celle de la symphonie. Le début de chaque chapitre annonce très brièvement et à la manière d'un pizzicato le thème qui est ensuite développé et repris dans une multitude de contextes différents pour aboutir à un finale dans lequel résonne tout ce qui précède.

Pour exprimer toutes ces dimensions multiples, Bloch disposait d'un matériau de choix : la langue allemande, extrêmement souple, malléable à souhait, et où l'abstrait et le concret se côtoient constamment avec bonheur, parfois même au sein d'un seul et même mot composé.

On peut dire que Bloch a saisi la quintessence de la langue allemande et en exploite à fond toutes les richesses. Son allemand est allemandissime.

Revenons maintenant à l'opération traduisante. Dans le cas du Principe Espérance, il s'agissait donc d'abord de saisir toutes ces dimensions citées plus haut; or, justement, en avoir une conscience toute conceptuelle à la manière du critique littéraire ne suffisait pas. Si le critique peut et même doit garder une certaine distance par rapport au texte qu'il analyse, le traducteur littéraire ne peut quant à lui se soustraire au processus d'identification. Plus cette identification avec la nature profonde de l'auteur et avec le monde mis en scène par lui est grande et plus le message aura de chances de passer dans la langue d'arrivée, car seul ce qui est ressenti, vécu de l'intérieur crée un effet d'authenticité, et le mot, le style, le ton justes viendront alors spontanément. En effet, la traduction d'un texte d'auteur ne peut en aucun cas être une imitation, ce doit être une reproduction, rnieux encore une re-création, et qui dit création dit inspiration, qui dit inspiration dit jaillissement spontané d'une source réelle.

Cette exigence s'avérait particulièrement impérieuse dans le cas de Bloch, dont la langue est avant tout affective et dont l'intention première est non pas tant de convaincre par le concept que de toucher, de faire ressentir et d'ébranler, conséquence directe des fondements de sa philosophie. C'est précisément grâce aux affinités électives profondes que j'avais avec l'auteur que j'ai pu consacrer tant d'années à traduire son enthousiasme, sa foi, son énergie, son courant chaud, sans courir le risque de me lasser et donc de perdre l'inspiration. C'est sans doute aussi grâce à une éducation musicale prolongée que je fus particulièrement réceptive au rythme et à la structure de son texte et que j'ai pu en tout cas les ressentir comme un mode d'expression qui m'était familier.

Bloch lui-même devait être conscient de l'importance du facteur d'empathie, car la toute première question qu'il $\mathrm{m}$ 'a posée lors de notre rencontre fut celle-ci : «Êtes-vous chrétienne ?» question qui d'abord m'a déconcertée, mais dont j'ai bientôt compris tout le poids. Ce qu'il lui importait de savoir, c'était si le traducteur de son Principe Espérance avait grandi dans la dimension messianique de l'espérance et ressenti de l'intérieur les valeurs qu'il mettait en lumière.

Bref, si l'énergie et l'enthousiasme de Bloch n'avaient été au départ une composante essentielle de ma nature profonde, je n'aurais jamais pu lui emboîter le pas pendant près de deux mille pages.

Je vous ai parlé jusqu'ici de l'empathie au niveau des rapports personnels entre l'auteur et le traducteur. Pourtant cette empathie existe aussi à un autre plan sur lequel le traducteur n'a, hélas, que très peu d'emprise: au plan de la confrontation de deux cultures.

En effet, la traduction n'implique ni plus ni moins que le passage d'une culture à une autre, par le truchement de la langue. La culture au sens large du terme, c'est le résultat bien concret d'un habitus de vie commune qui se manifeste dans la manière de penser, de réagir, et jusque dans les gestes ; c'est un organisme vivant qui a poussé lentement et 
qui se perpétue dans chaque être nouveau qui y naît, qui y baigne, qui y grandit. La langue, c'est en quelque sorte la peau de cette entité culturelle bien concrète. Aussi personnalisée que soit l'œuvre d'un auteur, elle reflète toujours, ne serait-ce que par réaction, son contexte culturel.

Pour en revenir à Ernst Bloch: il est d'origine juive, né en Allemagne et a passé la plus grande partie de son existence en exil, volontaire ou forcé. Et pourtant, rien n'est plus allemand que sa langue, car, qu'il le veuille ou non, cet instrument dont il se sert est le reflet de la culture allemande, c'est-à-dire d'une conception particulière du monde, d'une façon de voir les choses, de ressentir des catégories telles que le temps et l'espace: c'est le reflet d'une sensibilité à certaines choses ou au contraire de l'ignorance séculaire de certaines autres.

Ceci nous amène au problème complexe de la communication et, au bout du compte, de la traduction. Prenons pour exemple la conception anglo-saxonne du temps qui se reflète avec un riche éventail de nuances dans les temps de la conjugaison ou dans la forme progressive, cauchemar de nos étudiants francophones. Avant de l'appliquer correctement, il s'agit d'abord de changer de lunettes, de mettre sous la loupe ce que depuis des siècles on ignorait systématiquement, et même de considérer ses propres actes ou ceux du voisin sous un jour totalement différent: pour enfin arriver à comprendre par exemple que "you are silly» ne signifie pas la même chose que "you are being silly».

Pour illustrer d'un exemple amusant cet abîme qui se creuse parfois entre deux cultures, et qui est presque insurmontable linguistiquement, je voudrais vous raconter une anecdote qui m'a été rapportée. Un traducteur de l'allemand vers le français rencontre son homologue japonais: ils ont traduit le même livre dans lequel un terme allemand, schichtigkiekend, constitue une difficulté particulière de traduction. Ce participe présent signifie grosso modo «capable de percevoir les différentes couches, strates des choses et des êtres», autrement dit «perspicace ou sensible aux nuances». Notre culture occidentale, sensiblisée à l'analyse rationnelle, dispose au plan linguistique d'un nombre d'adjectifs suffisant pour rendre ce terme avec plus ou moins d'exactitude. À la question de savoir comment il l'avait traduit, le Japonais a répondu, d'ailleurs très fier de sa trouvaille : illuminé par Bouddha!

Pour en revenir à l'allemand : quelle mentalité se cache derrière cette langue et plus particulièrement derrière celle de Bloch?

En résumé, et comme le déclare d'ailleurs Nietzsche lui-même, les Allemands accordent au Devenir et à l'évolution plus de sens et de valeur qu'à l'Être, et ce sens profond de la dialectique transparaît dans le langage avec ce dynamisme et cette extraordinaire plasticité qui lui sont propres.

Au niveau lexical, l'allemand se distingue du français par son approche concrète des choses. Là où le français a déjà abstrait et en quelque sorte ne donne qu'une version décantée des phénomènes à l'aide de substantifs (la résignation, la grandeur, etc.), l'allemand colle souvent au concret, ce qui lui confère sa haute qualité métaphorique, symbolique et en fait un langage très vivant. C'est ainsi que pour décrire un phénomène ou une action, l'allemand dispose non seulement du substantif proprement dit, mais a de surcroît la faculté de tout substantiver: l'infinitif, l'adverbe, l'adjectif, le participe présent ou passé et même la conjonction! À côté du mot réalisation par exemple, l'allemand peut parler du réaliser, du réalisé ou du réalisant, mettant dans chaque cas l'accent sur un aspect particulier du phénomène général de la réalisation. Un autre exemple de plasticité et de souplesse de la langue allemande est sa faculté presque infinie de composer des mots. Ce que le mot composé allemand offre d'un coup, de façon globale et immédiate, sera rendu en français par un discours analytique. Le mot-tiroir allemand a pour effet de présenter le signifié comme une synthèse, ramassant des idées et permettant un 
maniement plus commode de notions complexes, mais aussi une concentration beaucoup plus intense de la phrase.

Au niveau de la syntaxe, la phrase allemande est souvent interminable, elle l'est d'autant plus qu'elle se veut précise, qu'elle se veut prédicat aussi complet et complexe que possible du sujet. Elle est réputée, souvent mal aimée des étrangers pour ses propositions incises dans lesquelles s'inscrivent d'autres incises et ainsi de suite, qui, non contentes de définir la face du sujet, en exposent aussi le verso, incluant déjà les contradictions et les oppositions. À la limite, la phrase allemande est en soi un petit débat, un choc des idées d'où doit jaillir la lumière, un serpent hégélien justement, où l'alpha, après avoir suivi tant de méandres, rejoint souvent son oméga dans une simple petite particule séparable qui rétablit, après bien des détours, le lien avec le corps du verbe situé en tête. Or, dans la traduction en français d'un tel matériau, que reste-t-il du génie de la langue allemande qui vient d'être décrit? Henri Arvon parle du «fossé infranchissable qui sépare la pensée allemande de la mentalité latine», ce qui nous ramène au problème de l'empathie à un niveau plus général: la mentalité française est-elle capable d'accueillir et d'exprimer la pensée allemande si intimement rendue dans la langue? Autrement dit: dispose-t-elle des outils linguistiques nécessaires pour restituer fidèlement la dimension allemande dans toutes ses nuances? Pour situer la question à un niveau plus imagé: une sculpture taillée dans le bois et inspirée par la matière du bois se laisserait-elle fidèlement reproduire dans le marbre? Évidemment non. Traduire, c'est bel et bien trahir. C'est écorcher vif une entité dans laquelle la forme et le contenu ne font qu'un. C'est lui arracher la peau qui était née de lui, pour lui en faire revêtir une autre qui collera plus ou moins bien. Dans le meilleur des cas et à ce niveau-ci, une bonne traduction sera celle qui masquera le plus habilement la blessure.

Ceci ramasse en quelques mots tout le problème de la traduction littéraire. Car, jusqu'où aller dans la transposition du texte original : quelle est «la limite au-delà de llaquelle votre traduction n'est plus valable»? Faut-il fournir un texte certes écrit avec des mots français, mais gardant toute la spécificité allemande, par exemple en forgeant des mots composés, en gardant la syntaxe complexe et lourde, en maintenant l'emploi abusif d'adverbes pour rester fidèle à la précision de l'original? Un tel texte sera illisible pour le francophone et on pourrait le comparer à un aliment pour lequel le lecteur ne dispose pas des sucs digestifs nécessaires.

Ou alors faut-il, autre extrême, franciser le texte original, c'est-à-dire lui appliquer les manies du français, donc l'approche française des choses, substituer par exemple l'élégance à la profondeur parfois un peu lourde de l'allemand, mais alors aussi substituer l'esprit français à l'esprit allemand et, en fin de compte, ne rien laisser subsister de la culture profonde du texte de départ? Pour exprimer une fois encore la chose de façon plus imagée: a-t-on le droit de faire revêtir à Faust le costume du gentilhomme français?

Je pourrais citer ici comme exemple célèbre la traduction par Gérard de Nerval du Faust de Goethe. Le texte français est superbe, sans aucun doute, mais on a beaucoup de peine à y reconnaître le Faust allemand; disons-le tout net: le texte de Gérard de Nerval est une géniale trahison.

Oui, et pourtant je conclurai ces considérations sur une note optimiste, parce que cet optimisme me semble pleinement justifié.

Tout d'abord l'empathie personnelle entre l'auteur et le traducteur, les affinités électives qui s'exercent aussi bien au niveau du genre, de la tonalité, de la sensibilité que des avatars biographiques ou du système de concepts offrent une première issue salvatrice pour venir à bout des obstacles interculturels. Malgré les divergences, les écarts parfois fondamentaux qui séparent deux cultures, deux individus, l'auteur et le traducteur trouvent un terrain d'entente grâce au magnétisme qui les rapproche; le traducteur se 
reconnaît dans l'auteur et cette dimension humaine commune est le premier pont qui permet de franchir les frontières et de surmonter les obstacles linguistiques. Dans son livre intitulé La Crise de la culture européenne, Rudolf Pannwitz donne lui aussi une réponse humaniste au problème et met en évidence les qualités d'ouverture vers l'autre. Il faut que les aillères tombent, que l'esprit de clocher s'évanouisse: Nos traductions, dit-il, même les meilleures, partent d'un mauvais principe: elles veulent "allemandiser» l'indien, le grec, l'anglais, au lieu d' «indianiser», de "grecquiser", d' «angliciser» l'allemand, car elles ont une vénération bien plus grande pour leurs propres habitudes langagières que pour le génie de la langue étrangère.

En effet, la tâche de traducteur consiste à élargir et à approfondir la langue-cible, la sienne, grâce aux apports de la langue étrangère, et c'est ici que réside sans doute la solution: manier correctement sa propre langue, certes, mais l'enrichir des nuances étrangères, lui ouvrir de nouveaux horizons, l'assouplir, la faire évoluer vers l'autre, sans pour autant, bien sûr, l'estropier et la dénaturer.

$\mathrm{Au}$ traducteur d'avoir la finesse nécessaire pour savoir où fixer le seuil. C'est une tâche d'enfantement ingrate et difficile, mais le texte qui naîtra alors ne sera, il est vrai, plus tout à fait écrit dans la langue pure et spontanée d'arrivée, le texte traduit sera une espèce de mutant issu d'un no man's land où deux cultures ont heureusement fusionné, grâce à l'entremise du traducteur qui, à la limite idéale, devient alors le porteur d'un message d'ouverture, de générosité, de tolérance et d'entente et donc, finalement, de paix. 